\title{
北京东灵山3种温带森林土壤呼吸及其20年的变化
}

\author{
姚 辉 胡雪洋 朱江玲 朱剑霄 吉成均 方精云
}

北京大学城市与环境学院生态学系, 北京大学地表过程分析与模拟教育部重点实验室, 北京 100871

摘 要 土壤呼吸是陆地生态系统最主要的碳释放过程。为了探讨温带森林土壤呼吸在长时间尺度的变化, 利用北京东灵山 地区的白桦(Betula platyphylla)林、辽东栋(Quercus wutaishanica)林和油松(Pinus tabuliformis)林3种温带森林永久样地, 于 2012-2015年对其土壤呼吸进行测定, 并与1994-1995年的测定结果进行了比较。结果显示: 2012-2015年, 白桦林的平均年土 壤呼吸量为 $(574 \pm 21) \mathrm{g} \mathrm{C} \cdot \mathrm{m}^{-2} \cdot \mathrm{a}^{-1}$, 显著高于辽东栋林 $(455 \pm 31) \mathrm{g} \mathrm{C} \cdot \mathrm{m}^{-2} \cdot \mathrm{a}^{-1}$ 和油松林 $(414 \pm 35) \mathrm{g} \mathrm{C} \cdot \mathrm{m}^{-2} \cdot \mathrm{a}^{-1}$, 比20年前 (1994-1995年)的估测值分别增加了 85\%、17\%和73\%。这些结果表明, 近20年来这3种生态系统的碳周转速率明显加快。

关键词 土壤呼吸, $5 \mathrm{~cm}$ 土壤温度, 温度敏感性系数 $\left(Q_{10}\right)$, 年通量, 温带森林

引用格式: 姚辉, 胡雪洋, 朱江玲, 朱剑霄, 吉成均, 方精云 (2015). 北京东灵山3种温带森林土壤呼吸及其20年的变化. 植物生态学报, 39, 849-856. doi: 10.17521/cjpe.2015.0081

\section{Soil respiration and the 20-year change in three temperate forests in Mt. Dongling, Beijing}

YAO Hui, HU Xue-Yang, ZHU Jiang-Ling, ZHU Jian-Xiao, JI Cheng-Jun, and FANG Jing-Yun*

Department of Ecology, College of Urban and Environmental Sciences, and Key Laboratory for Earth Surface Processes of the Ministry of Education, Peking University, Beijing 100871, China

\section{Abstract}

Aims Our objective was to explore changes in soil respiration of three temperate forests in Mt. Dongling, Beijing over the last 20 years from the year of 1994-1995 to 2012-2015.

Methods We re-investigated the permanent plots of three temperate forests (Betula platyphylla forest, Quercus wutaishanica forest and Pinus tabuliformis forest) which were established in 1992. We measured soil respiration for 3 years (2012-2015) using a LI-8100 Soil Respiration System. Continuous soil temperatures at $5 \mathrm{~cm}$ depth were measured at the same time. Annual soil respiration was accumulated using a relationship between soil respiration and soil temperature .

Important findings We found that soil respiration rates were significantly correlated with soil temperature at 5 $\mathrm{cm}$ depth and that these correlations differed remarkably among the three forests. Annual soil respiration in $B$. platyphylla forest was highest, with a 3-year average of $(574 \pm 21) \mathrm{g} \mathrm{C} \cdot \mathrm{m}^{-2}$, followed by Q. wutaishanica forest

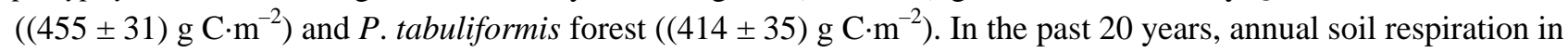
all these forests increased significantly: compared to 1994-1995, the average in 2012-2015 increased by 85\%, $17 \%$ and $73 \%$ for B. platyphylla, Q. wutaishanica, and P. tabuliformis forests, respectively.

Key words soil respiration, soil temperature at $5 \mathrm{~cm}$ depth, temperature sensitivity $\left(Q_{10}\right)$, annual flux, temperate forest

Citation: Yao H, Hu XY, Zhu JL, Zhu JX, Ji CJ, Fang JY (2015). Soil respiration and the 20-year change in three temperate forests in Mt. Dongling, Beijing. Chinese Journal of Plant Ecology, 39, 849-856. doi: 10.17521/cjpe. 2015.0081

土壤呼吸是陆地生态系统碳循环的重要组分。 全球土壤呼吸年通量达60-110 Pg C (Raich \& Schlesinger, 1992; Raich \& Potter, 1995; Schlesinger \& Andrews, 2000; 方精云和王娓, 2007; BondLamberty \& Thomson, 2010)。在全球变化背景下,
土壤呼吸的微小变化就可能对大气 $\mathrm{CO}_{2}$ 浓度产生重 大影响(Schlesinger \& Andrews, 2000; Davidson \& Janssens, 2006; Bronson et al., 2008)。影响土壤呼吸 的主要因素包括底物供应(Högberg et al., 2001, 2009)、土壤温度(Atkin et al., 2000; Rayment \&

收稿日期Received: 2015-06-03 接受日期Accepted: 2015-06-20

* 通讯作者Author for correspondence (E-mail: jyfang@urban.pku.edu.cn) 
Jarvis, 2000; Davidson \& Janssens, 2006)、土壤水分 (Bowden et al., 1993)、养分供给(Burton et al., 1998)、 土壤pH值(Sitaula et al., 1995)和微生物活性(Fisk \& Fahey, 2001)等。其中, 土壤温度和水分是最直接的 影响因子, 在土壤温度高、水分充足的区域, 土壤呼 吸相对较高。

由于缺乏长期的定位观测, 现有的土壤呼吸研 究的时间尺度大多较短(Savage \& Davidson, 2001), 在国内较长时间的研究更是少见。Fang等(2007)于 1992年在北京东灵山的白华(Betula platyphylla)林、 辽东栋(Quercus wutaishanica)林和油松(Pinus tabuliformis)林3种典型温带森林中, 各建立了一块永久 样地, 1994-1995年分别测定了其土壤呼吸速率, 并 估算了其年通量(刘绍辉等, 1998)。近20年来土壤呼 吸是否发生了变化? 为探明这个问题, 我们于 2012-2015年采用与 20年前同样的方法(刘绍辉, 1998; Fang et al., 2007)对3块样地的土壤呼吸进行 了复查观测。

\section{1 材料和方法}

\section{1 研究区概况}

本研究区位于中国科学院北京森林生态系统定 位研究站 $\left(39.97^{\circ} \mathrm{N}, 115.43^{\circ} \mathrm{E}\right)$ 。该地区属于温带半 湿润季风气候, 四季分明, 夏季高温多雨, 冬季寒 冷干燥。年降水量为 $(570.3 \pm 112.2) \mathrm{mm}$, 大约 $63 \%$ 集中在6-8月份; 年平均气温为 $(4.9 \pm 0.6){ }^{\circ} \mathrm{C}$, 最热 的7月份平均气温为 $(18.7 \pm 1.0){ }^{\circ} \mathrm{C}$, 最冷的1月份平 均气温为 $(-10.4 \pm 1.5){ }^{\circ} \mathrm{C}$ (苏宏新和李广起, 2012)。 现有植被以天然的落叶阔叶次生林(白桦林和辽东 栎林)和人工针叶林(油松林)为主。

\section{2 样地情况}

依据1992年对树木所做的标记以及文献(Fang et al., 2007)记录, 2011年夏季我们对白桦林、辽东栋 林和油松林的永久样地进行了复查。

白桦林样地 $\left(39.95^{\circ} \mathrm{N}, 115.43^{\circ} \mathrm{E}\right)$ 位于海拔 $1350 \mathrm{~m}$ 的西北向山坳阴坡, 阴暗潮湿。样地大小为 $30 \mathrm{~m} \times 40 \mathrm{~m}$, 乔木层主要为白桦, 伴有少量的黑桦 (Betula dahurica)和山杨(Populus davidiana); 乔木 亚层主要包含五角枫(Acer pictum subsp. mono)和北 京花楸(Sorbus discolor)等; 灌木层十分茂盛, 主要 有毛榛(Corylus mandshurica)、巧玲花(Syringa pubescens)、六道木(Zabelia biflora)和土庄绣线菊
(Spiraea pubescens) 等; 草本层茂密。土层厚90$100 \mathrm{~cm}$ 。

辽东栋林样地 $\left(39.96^{\circ} \mathrm{N}, 115.42^{\circ} \mathrm{E}\right)$ 位于山腰西 南向的阳坡上，海拔 $1250 \mathrm{~m}$ 。样地大小为 $30 \mathrm{~m} \times 40$ $\mathrm{m}$, 乔木层主要包括辽东栋, 伴有少量黑桦; 乔木 亚层主要由色木槭和花曲柳(Fraxinus chinensis subsp. rhynchophylla)构成; 林下灌木茂盛, 常见物 种包括六道木、土庄绣线菊、大花潰疏(Deutzia grandiflora)、小花溲疏(Deutzia parviflora)、胡枝子 (Lespedeza bicolor) 等; 草本植物繁茂。土层厚 $90-120 \mathrm{~cm}$ 。

油松林样地 $\left(39.96^{\circ} \mathrm{N}, 115.43^{\circ} \mathrm{E}\right)$ 位于山脚东南 向的阳坡上, 海拔 $1150 \mathrm{~m}$, 林龄为 50 a左右。样地大 小为 $20 \mathrm{~m} \times 50 \mathrm{~m}$, 其林相整齐, 灌木层及草本层植 物稀少。土层厚100-110 cm。

\section{3 土壤呼吸测定和年通量估算}

在每个样地均匀布设 10 个固定的直径 $20 \mathrm{~cm}$ 的 Collar环, 进行土壤呼吸测定。Collar环插入土壤约 5 $\mathrm{cm}$, 用剪刀将Collar环中的杂草剪净。

从2012年开始, 于生长季(5-9月)每月中旬, 选 择天气晴好的白天, 采用LI-8100A土壤 $\mathrm{CO}_{2}$ 通量自 动测定系统(LI-COR, Lincoln, USA)测定土壤呼吸, 其自带的温度探头同时记录地下 $5 \mathrm{~cm}$ 土壤温度。测 定时间为每日9:00-15:00。

从2012年 4 月开始, 在每种森林类型地下 $5 \mathrm{~cm}$ 各放置一个StowAway TidbiT土壤温度记录仪(Onset Computer Corp., Bourne, USA), 连续记录土壤温度, 采样间隔为 $1 \mathrm{~h}$ 。

土壤呼吸速率与地下 $5 \mathrm{~cm}$ 土壤温度的拟合方程 为:

$$
S R=a \cdot e^{b \cdot T}
$$

$S R$ 为土壤呼吸速率 $\left(\mu \mathrm{mol} \mathrm{CO} \mathrm{CO}_{2} \cdot \mathrm{m}^{-2} \cdot \mathrm{s}^{-1}\right), T$ 为地下 $5 \mathrm{~cm}$ 深处的土壤温度, $a 、 b$ 为拟合参数, 其中, $a$ 表示 土壤基础呼吸速率 $\left(R_{0}\right)$ 。

采用如下推导公式计算温度敏感性系数 $\left(Q_{10}\right)$ :

$Q_{10}=\mathrm{e}^{10 \cdot b}$

$Q_{10}$ 表示温度每升高 $10{ }^{\circ} \mathrm{C}$ 时土壤呼吸的变化倍 数; $b$ 为式(1)中的 $b$ 。

全年土壤 $\mathrm{CO}_{2}$ 通量用温度拟合法获取: 首先建 立土壤呼吸速率与地下 $5 \mathrm{~cm}$ 土壤温度的指数关系, 然后利用TidbiT记录的 $5 \mathrm{~cm}$ 土壤温度连续监测数据, 累加全年的呼吸值, 计算各样地全年土壤 $\mathrm{CO}_{2}$ 通量 
$\left(\right.$ g C $\left.\cdot m^{-2} \cdot a^{-1}\right)$ 。

\section{2 结果}

\section{1 土壤呼吸与 $5 \mathrm{~cm}$ 土壤温度的关系}

在白桦林、辽东栋林和油松林中, 土壤呼吸速率 与 $5 \mathrm{~cm}$ 土壤温度均呈显著的指数相关关系, 其拟合 度 $\left(R^{2}\right)$ 分别为 $0.68 、 0.78$ 和 $0.68(p<0.001$, 图1)。三个 样地土壤呼吸速率与温度的相关性也存在显著差异, 其中白桦林的土壤基础呼吸速率 $\left(R_{0}\right)$ 最高, 为 $(0.51 \pm$ 0.05) $\mu \mathrm{mol} \cdot \mathrm{m}^{-2} \cdot \mathrm{s}^{-1}$, 显著高于辽东栋林和油松林 (分别为 $(0.29 \pm 0.04)$ 和 $(0.20 \pm 0.04) \mu \mathrm{mol} \cdot \mathrm{m}^{-2}$. $\left.\mathrm{s}^{-1}\right)$ 。辽东栎林 $Q_{10}$ 最高, 为 $4.13 \pm 0.37$; 油松林次之, 为 $3.97 \pm 0.47$; 白桦林最低, 为 $3.69 \pm 0.29(p<0.05$, 表1)。

\section{2 土壤呼吸的季节变化}

三个样地的土壤呼吸均呈单峰曲线的季节变化 规律(图2)。利用 $Q_{10}$ 模型估算的白桦林土壤呼吸速 率的范围在 $0.13-6.50 \mu \mathrm{mol} \cdot \mathrm{m}^{-2} \cdot \mathrm{s}^{-1}$ 之间, 平均值为 $1.52 \mu \mathrm{mol} \cdot \mathrm{m}^{-2} \cdot \mathrm{s}^{-1} ; 1$ 月份土壤呼吸速率最低, 随着土 壤温度的回升不断增加, 在7月份达到最高值。辽东 栋林土壤呼吸速率范围为 $0.07-5.45 \mu \mathrm{mol} \cdot \mathrm{m}^{-2} \cdot \mathrm{s}^{-1}$,
平均值为 $1.20 \mu \mathrm{mol} \cdot \mathrm{m}^{-2} \cdot \mathrm{s}^{-1}$ 。油松林土壤呼吸速率在 0.03-4.85 $\mu \mathrm{mol} \cdot \mathrm{m}^{-2} \cdot \mathrm{s}^{-1}$ 之间, 平均值为 $1.09 \mu \mathrm{mol}$. $\mathrm{m}^{-2} \cdot \mathrm{s}^{-1}$ 。

\section{3 土壤呼吸年通量及 20 年的变化}

根据土壤呼吸速率与土壤表层 $5 \mathrm{~cm}$ 温度关系的 $Q_{10}$ 模型，分别推算2012-2015年间3种森林的土壤 呼吸年通量。结果显示, 白桦林的平均年通量为

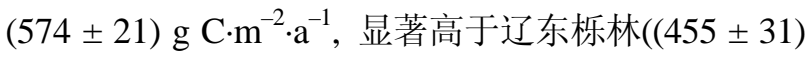
$\left.\mathrm{g} \mathrm{C} \cdot \mathrm{m}^{-2} \cdot \mathrm{a}^{-1}\right)$ 和油松林 $\left((414 \pm 35) \mathrm{g} \mathrm{C} \cdot \mathrm{m}^{-2} \cdot \mathrm{a}^{-1}\right)$ (图3)。

如前所述, 采用相似的土壤呼吸测定方法, 刘 绍辉等(1998)于1994-1995年对3块样地的土壤呼吸 进行了测定。白桦林、辽东栋林和油松林的土壤呼 吸的年通量分别为 $310 、 390$ 和 $240 \mathrm{~g} \mathrm{C} \cdot \mathrm{m}^{-2} \cdot \mathrm{a}^{-1}$ 。20年 来，3种森林的土壤呼吸年通量分别增加了 $85 \%$ 、 $17 \%$ 和 $73 \%$ 。

\section{3 讨论}

\section{1 不同森林土壤呼吸的比较}

温度是影响土壤呼吸速率的重要因素。在本研 究中, 白桦林的海拔相对最高, $5 \mathrm{~cm}$ 深度的土壤年 平均温度最低 $\left((4.55 \pm 0.25){ }^{\circ} \mathrm{C}\right)$, 但其土壤呼吸的年

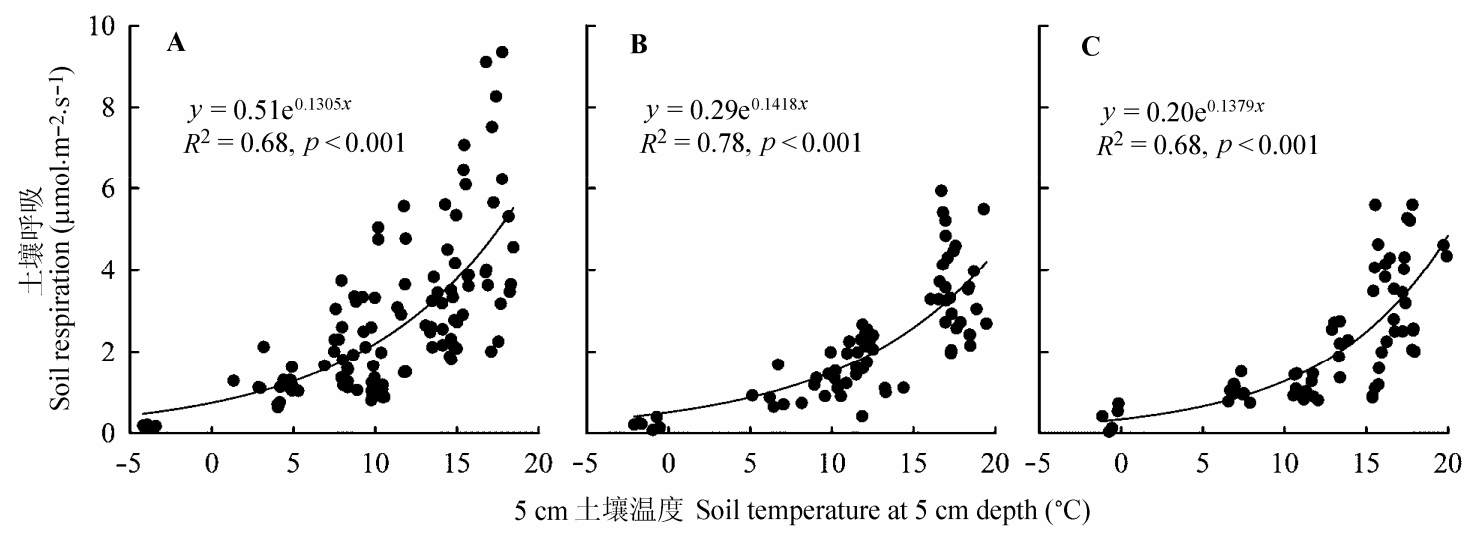

图1 北京东灵山3种温带森林土壤呼吸与 $5 \mathrm{~cm}$ 土壤温度之间的关系。 $\mathbf{A}$, 白华林。 B, 辽东栋林。C, 油松林。

Fig. 1 Relationships between soil respiration and soil temperature at $5 \mathrm{~cm}$ depth in three temperate forests on Mt. Dongling, Beijing. A, Betula platyphylla forest. B, Quercus wutaishanica forest. C, Pinus tabuliformis forest.

表1 北京东灵山3种温带森林的基础土壤呼吸 $\left(R_{0}, \mu \mathrm{mol} \cdot \mathrm{m}^{-2} \cdot \mathrm{s}^{-1}\right)$ 和温度敏感性系数 $\left(Q_{10}\right)$

Table 1 Fundamental soil respiration $\left(R_{0}, \mu \mathrm{mol} \cdot \mathrm{m}^{-2} \cdot \mathrm{s}^{-1}\right)$ and temperature sensitivity $\left(Q_{10}\right)$ in three temperate forests on Mt. Dongling, Beijing

\begin{tabular}{lcc}
\hline 样地类型 Forest type & 基础呼吸速率 Fundamental soil respiration $\left(R_{0}\right)$ & $Q_{10}$ 值 Temperature sensitivity \\
\hline 白桦林 Betula platyphylla forest & $0.51(0.05)^{\mathrm{a}}$ & $3.69(0.29)^{\mathrm{a}}$ \\
辽东栋林 Quercus wutaishanica forest & $0.29(0.04)^{\mathrm{b}}$ & $4.13(0.37)^{\mathrm{b}}$ \\
油松林 Pinus tabuliformis forest & $0.20(0.04)^{\mathrm{c}}$ & $3.97(0.47)^{\mathrm{c}}$ \\
\hline
\end{tabular}

a, b, c, 显著性字母标记 $(\alpha=0.05)$ 。括号内数字表示标准偏差。

a, b, c, significance alphabetic flag $(\alpha=0.05)$. Figures in parentheses indicate standard deviation. 


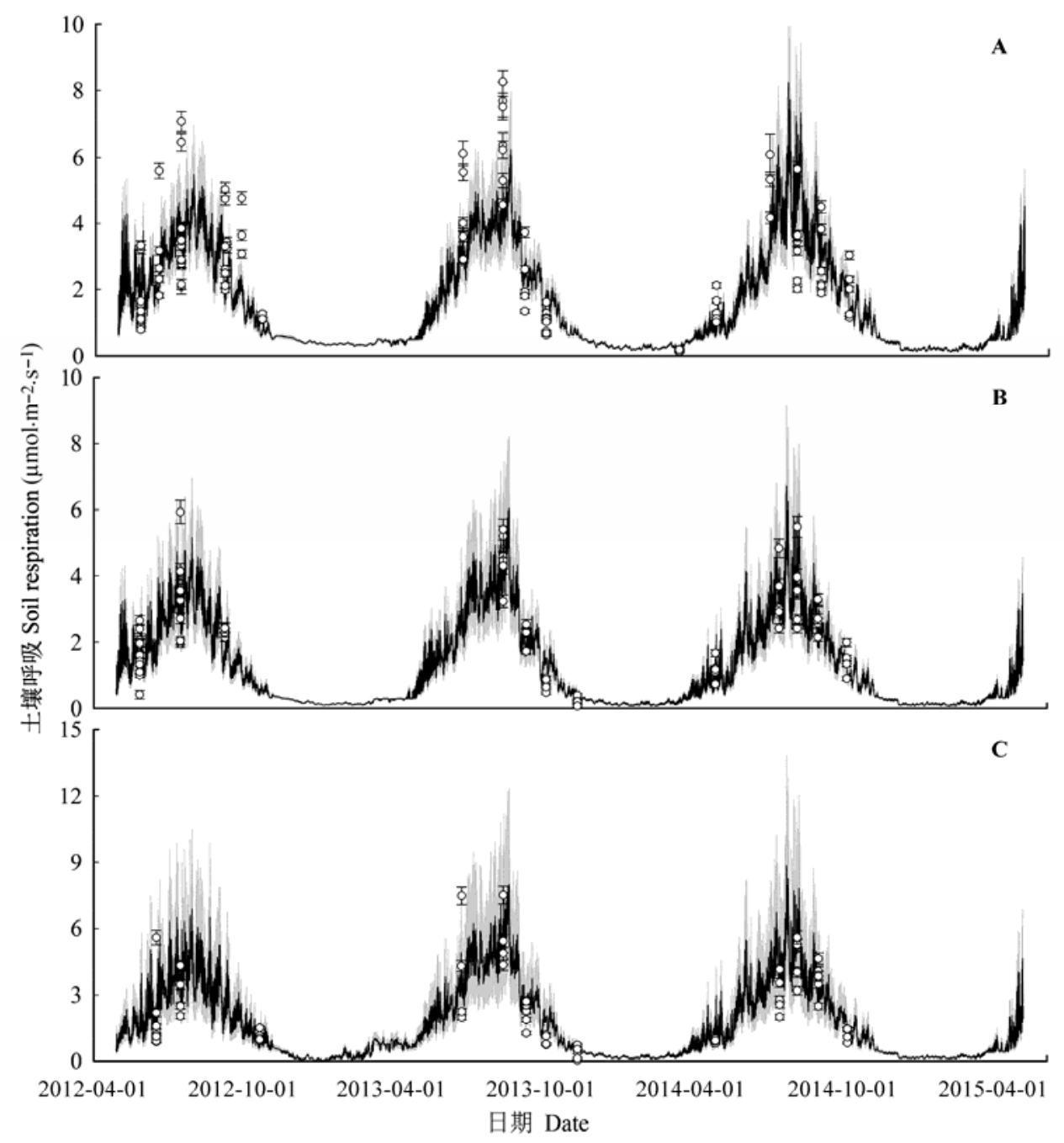

图2 北京东灵山3种温带森林拟合(实线)和实测(点)的土壤呼吸随时间的变化。A, 白桦林。 $\mathbf{B}$, 辽东栋林。 $\mathbf{C}$, 油松林。

Fig. 2 Temporal patterns of model fitted (line) and measured (point) soil respiration in three temperate forests on Mt. Dongling, Beijing. A, Betula platyphylla forest. B, Quercus wutaishanica forest. C, Pinus tabuliformis forest.

释放量却最高。这一结果可能暗示：除温度外, 其 他因素也影响着土壤呼吸, 如土壤底物是呼吸速率 的重要影响因素之一(Högberg et al., 2001, 2009)。在 我们的研究中, 白桦林表层土壤有机质含量在3个 样地中是最高的(表层20 $\mathrm{cm}$ 土壤碳密度为 $83.8 \mathrm{Mg}$ $\left.\mathrm{C} \cdot \mathrm{hm}^{-2}\right)$, 油松林次之 $\left(75.1 \mathrm{Mg} \mathrm{C} \cdot \mathrm{hm}^{-2}\right)$, 辽东栋林最 低(71.8 Mg C· $\mathrm{hm}^{-2}$ )(胡雪洋, 2015), 这可能导致白 桦林的土壤基础呼吸速率 $\left((0.51 \pm 0.05) \mu \mathrm{mol} \cdot \mathrm{m}^{-2}\right.$. $\left.\mathrm{s}^{-1}\right)$ 显著高于辽东栋林 $\left((0.29 \pm 0.04) \mu \mathrm{mol} \cdot \mathrm{m}^{-2} \cdot \mathrm{s}^{-1}\right)$ 和 油松林 $\left((0.20 \pm 0.04) \mu \mathrm{mol} \cdot \mathrm{m}^{-2} \cdot \mathrm{s}^{-1}\right)$ (表1)。另外, 土壤 水分通过影响底物和 $\mathrm{O}_{2}$ 的扩散而调控呼吸作用 (Linn \& Doran, 1984)。已有研究表明, 土壤呼吸速 率随年降水量的增加呈线性增长(Raich \& Schlesinger, 1992)。受地形影响, 白桦林样地地势平坦且水 www.plant-ecology.com
分条件良好，有利于土壤呼吸，而油松林地处西南 坡, 光照强, 林下植被少, 保水性能差(刘绍辉等, 1998), 这可能使土壤呼吸受到限制。

为了与温带地区其他森林的土壤呼吸进行比较, 我们搜集了国内外温带森林土壤呼吸的相关文献(表 2)。结果发现, 温带阔叶林的土壤呼吸年通量为464$1105 \mathrm{~g} \mathrm{C} \cdot \mathrm{m}^{-2} \cdot \mathrm{a}^{-1}$, 平均值为739 $\mathrm{g} \mathrm{C} \cdot \mathrm{m}^{-2} \cdot \mathrm{a}^{-1}$, 高于我 们的测定值, 即便是年通量最大的白桦林 $574 \mathrm{~g}$ $\left.\mathrm{C} \cdot \mathrm{m}^{-2} \cdot \mathrm{a}^{-1}\right)$ 也处于表 2 中的较低水平。相对于表 2 中的 温带针叶林土壤呼吸年通量(403-1 $300 \mathrm{~g} \mathrm{C} \cdot \mathrm{m}^{-2} \cdot \mathrm{a}^{-1}$, 平均值620 $\left.\mathrm{g} \mathrm{C} \cdot \mathrm{m}^{-2} \cdot \mathrm{a}^{-1}\right)$, 本研究中的油松林也处于 较低水平 $\left(413 \mathrm{~g} \mathrm{C} \cdot \mathrm{m}^{-2} \cdot \mathrm{a}^{-1}\right)$ 。这可能是由于早期研究 多用碱吸收法, 高估了呼吸速率(方精云等, 1995; Grogan \& Chapin III, 1999)。 
表2 温带森林年土壤呼吸量 $\left(\mathrm{g} \mathrm{C} \cdot \mathrm{m}^{-2} \cdot \mathrm{a}^{-1}\right)$

Table 2 Annual soil respiration of temperate forests $\left(\mathrm{g} \mathrm{C}^{-2} \cdot \mathrm{m}^{-2} \cdot \mathrm{a}^{-1}\right)$

\begin{tabular}{|c|c|c|c|c|}
\hline $\begin{array}{l}\text { 地点 } \\
\text { Locality }\end{array}$ & $\begin{array}{l}\text { 经纬度 } \\
\text { Location }\end{array}$ & $\begin{array}{l}\text { 主要树种 } \\
\text { Dominant species }\end{array}$ & $\begin{array}{l}\text { 年呼吸量1) } \\
\text { Annual soil } \\
\text { respiration }\end{array}$ & $\begin{array}{l}\text { 文献来源 } \\
\text { Reference }\end{array}$ \\
\hline \multicolumn{5}{|l|}{ 温带阔叶林 Temperate broad-leaved forests } \\
\hline 英国肯特郡 Kent, England & $51.33^{\circ} \mathrm{N}, 1.08^{\circ} \mathrm{E}$ & Castanea sativa & $630^{\mathrm{a}}$ & Anderson, 1973 \\
\hline 英国肯特郡 Kent, England & $51.33^{\circ} \mathrm{N}, 1.08^{\circ} \mathrm{E}$ & Fagus sylvatica & $575^{\mathrm{a}}$ & Anderson, 1973 \\
\hline 德国海德堡 Heidelberg, Germany & $49.33^{\circ} \mathrm{N}, 8.67^{\circ} \mathrm{E}$ & 水青冈 Fagus spp., 云杉 Picea spp. & $470^{\mathrm{a}}$ & Dörr \& Münnich, 1987 \\
\hline 美国华盛顿州 Washington, USA & $47.38^{\circ} \mathrm{N}, 121.95^{\circ} \mathrm{W}$ & Alnus rubra & $565^{\mathrm{a}}$ & Vogt et al., 1980 \\
\hline 美国明尼苏达州 Minnesota, USA & $45.50^{\circ} \mathrm{N}, 93.17^{\circ} \mathrm{W}$ & 栋 Quercus spp. & $794^{\mathrm{b}}$ & Reiners, 1968 \\
\hline 美国明尼苏达州 Minnesota, USA & $45.50^{\circ} \mathrm{N}, 93.17^{\circ} \mathrm{W}$ & 梣 Fraxinus spp. & $707^{\mathrm{b}}$ & Reiners, 1968 \\
\hline 美国明尼苏达州 Minnesota, USA & $45.50^{\circ} \mathrm{N}, 93.17^{\circ} \mathrm{W}$ & 崖柏 Thuja spp., 桦木 Betula spp. & $739^{\mathrm{b}}$ & Reiners, 1968 \\
\hline 美国密苏里州 Missouri, USA & $38.80^{\circ} \mathrm{N}, 92.20^{\circ} \mathrm{W}$ & 栋 Quercus spp., 山核桃 Carya spp. & $1013^{\mathrm{b}}$ & Garrett \& Cox, 1973 \\
\hline 美国田纳西州 Tennessee, USA & $35.97^{\circ} \mathrm{N}, 84.28^{\circ} \mathrm{W}$ & Lilriodendron tulipifera & $1065^{\mathrm{b}}$ & Edwards \& Harris, 1977 \\
\hline 美国田纳西州 Tennessee, USA & $35.97^{\circ} \mathrm{N}, 84.28^{\circ} \mathrm{W}$ & Lilriodendron tulipifera & $505^{\mathrm{b}}$ & $\begin{array}{l}\text { Edwards \& Ross-Todd, } \\
1979\end{array}$ \\
\hline 美国田纳西州 Tennessee, USA & $35.97^{\circ} \mathrm{N}, 84.28^{\circ} \mathrm{W}$ & 栋 Quercus spp., 山核桃 Carya spp. & $529^{\mathrm{b}}$ & $\begin{array}{l}\text { Edwards \& Ross-Todd, } \\
1983\end{array}$ \\
\hline 美国田纳西州 Tennessee, USA & $35.97^{\circ} \mathrm{N}, 84.28^{\circ} \mathrm{W}$ & Quercus prinus & $610^{\mathrm{b}}$ & Johnson \& van Hook, 1989 \\
\hline 美国北卡罗来纳州 North Carolina, USA & $35.05^{\circ} \mathrm{N}, 83.42^{\circ} \mathrm{W}$ & 栋 Quercus spp. & $857^{\mathrm{b}}$ & Johnson \& van Hook, 1989 \\
\hline 中国河南 Henan, China & $33.47^{\circ} \mathrm{N}, 111.92^{\circ} \mathrm{E}$ & 檞栋 Quercus aliena & $1105^{\mathrm{c}}$ & Chang et al., 2007 \\
\hline 中国河南 Henan, China & $33.47^{\circ} \mathrm{N}, 111.92^{\circ} \mathrm{E}$ & 葪栋 Quercus aliena & $779^{\mathrm{c}}$ & Chang et al., 2007 \\
\hline 中国河南 Henan, China & $33.47^{\circ} \mathrm{N}, 111.92^{\circ} \mathrm{E}$ & $\begin{array}{l}\text { 䚞栋 Quercus aliena, } \\
\text { 栓皮栋 Quercus variabilis }\end{array}$ & $912^{\mathrm{c}}$ & Chang et al., 2007 \\
\hline 中国河南 Henan, China & $33.47^{\circ} \mathrm{N}, 111.92^{\circ} \mathrm{E}$ & 栓皮栋 Quercus variabilis & $900^{\mathrm{c}}$ & Chang et al., 2007 \\
\hline 中国陕西 Shaanxi, China & $33.38^{\circ} \mathrm{N}, 108.50^{\circ} \mathrm{E}$ & 槲栋 Quercus aliena & $771^{\mathrm{a}}$ & Liu et al., 2003 \\
\hline 中国黑龙江 Heilongjiang, China & $45.37^{\circ} \mathrm{N}, 127.53^{\circ} \mathrm{E}$ & 蒙古栎 Quercus mongolica & $785^{\mathrm{c}}$ & Wang et al., 2006 \\
\hline 中国黑龙江 Heilongjiang, China & $45.37^{\circ} \mathrm{N}, 127.53^{\circ} \mathrm{E}$ & $\begin{array}{l}\text { 山杨 Populus davidiana, } \\
\text { 白华 Betula platyphylla }\end{array}$ & $813^{\mathrm{c}}$ & Wang et al., 2006 \\
\hline 中国黑龙江 Heilongjiang, China & $45.37^{\circ} \mathrm{N}, 127.53^{\circ} \mathrm{E}$ & $\begin{array}{l}\text { 山杨 Populus davidiana, } \\
\text { 胡桃楸 Juglans mandshurica }\end{array}$ & $786^{\mathrm{c}}$ & Wang et al., 2006 \\
\hline 中国黑龙江 Heilongjiang, China & $45.37^{\circ} \mathrm{N}, 127.53^{\circ} \mathrm{E}$ & 水曲柳 Fraxinus mandshurica & $781^{\mathrm{C}}$ & Wang et al., 2006 \\
\hline 中国河北 Heibei, China & $42.50^{\circ} \mathrm{N}, 117.35^{\circ} \mathrm{E}$ & 白桦 Betula platyphylla & $575^{\mathrm{d}}$ & Wang et al., 2010 \\
\hline 中国北京 Beijing, China & $39.95^{\circ} \mathrm{N}, 115.42^{\circ} \mathrm{E}$ & 白桦 Betula platyphylla & $574^{\mathrm{d}}$ & 本研究 This study \\
\hline 中国北京 Beijing, China & $39.95^{\circ} \mathrm{N}, 115.42^{\circ} \mathrm{E}$ & 辽东栋 Quercus wutaishanica & $455^{\mathrm{d}}$ & 本研究 This study \\
\hline \multicolumn{5}{|l|}{ 温带针叶林 Temperate coniferous forests } \\
\hline 美国华盛顿州 Washington, USA & $47.38^{\circ} \mathrm{N}, 121.95^{\circ} \mathrm{W}$ & 花旗松 Pseudotsuga menziesii & $490^{\mathrm{a}}$ & Vogt et al., 1980 \\
\hline 美国华盛顿州 Washington, USA & $47.38^{\circ} \mathrm{N}, 121.95^{\circ} \mathrm{W}$ & Tsuga heterophylla & $650^{\mathrm{a}}$ & Vogt et al., 1980 \\
\hline 美国华盛顿州 Washington, USA & $47.32^{\circ} \mathrm{N}, 121.58^{\circ} \mathrm{W}$ & Abies amabilis & $620^{\mathrm{a}}$ & Vogt et al., 1980 \\
\hline 美国南卡罗来纳州 South Carolina, USA & $33.50^{\circ} \mathrm{N}, 81.67^{\circ} \mathrm{W}$ & 长叶松 Pinus palustris & $509^{\mathrm{a}}$ & Reinke et al., 1981 \\
\hline 美国佛罗里达州 Florida, USA & $30^{\circ} \mathrm{N}, 82^{\circ} \mathrm{W}$ & 湿地松 Pinus elliottii & $1300^{\mathrm{b}}$ & Ewel et al., 1987a \\
\hline 美国佛罗里达州 Florida, USA & $30^{\circ} \mathrm{N}, 82^{\circ} \mathrm{W}$ & 湿地松 Pinus elliottii & $850^{\mathrm{b}}$ & Ewel et al., 1987b \\
\hline 中国陕西 Shaanxi, China & $33.38^{\circ} \mathrm{N}, 108.50^{\circ} \mathrm{E}$ & 油松 Pinus tabuliformis & $609^{\mathrm{a}}$ & Liu et al., 2003 \\
\hline 中国甘肃 Gansu, China & $38.43^{\circ} \mathrm{N}, 99.90^{\circ} \mathrm{E}$ & 青海云杉 Picea crassifolia & $584^{c}$ & Chang et al., 2008 \\
\hline 中国甘肃 Gansu, China & $38.43^{\circ} \mathrm{N}, 99.90^{\circ} \mathrm{E}$ & 华北落叶松 Larix principis-rupprechtii & $570^{\mathrm{c}}$ & Chang et al., 2008 \\
\hline 中国甘肃 Gansu, China & $38.43^{\circ} \mathrm{N}, 99.90^{\circ} \mathrm{E}$ & 祁连圆柏 Sabina przewalskii & $518^{\mathrm{c}}$ & Chang et al., 2008 \\
\hline 中国黑龙江 Heilongjiang, China & $45.37^{\circ} \mathrm{N}, 127.53^{\circ} \mathrm{E}$ & 红松 Pinus koraiensis & $514^{\mathrm{c}}$ & Wang et al., 2006 \\
\hline 中国黑龙江 Heilongjiang, China & $45.37^{\circ} \mathrm{N}, 127.53^{\circ} \mathrm{E}$ & 兴安落叶松 Larix gmelinii & $403^{c}$ & Wang et al., 2006 \\
\hline 中国河北 Heibei, China & $42.50^{\circ} \mathrm{N}, 117.35^{\circ} \mathrm{E}$ & 欧洲赤松 Pinus sylvestris & $453^{\mathrm{d}}$ & Wang et al., 2010 \\
\hline 中国河北 Heibei, China & $42.50^{\circ} \mathrm{N}, 117.35^{\circ} \mathrm{E}$ & 华北落叶松 Larix principis-rupprechtii & $435^{\mathrm{d}}$ & Wang et al., 2010 \\
\hline 中国北京 Beijing, China & $39.95^{\circ} \mathrm{N}, 115.42^{\circ} \mathrm{E}$ & 油松 Pinus tabuliformis & $414^{\mathrm{d}}$ & 本研究 This study \\
\hline
\end{tabular}

1)不同字母表示土壤呼吸的测定方法: a, 碱吸收法; b, 红外气体分析仪; c, LI-6400; d, LI-8100。

1) Different letters denote the different measurements of soil respiration: a, alkali absorption method; b, infrared gas analyzer; c, LI-6400; d, LI-8100. 


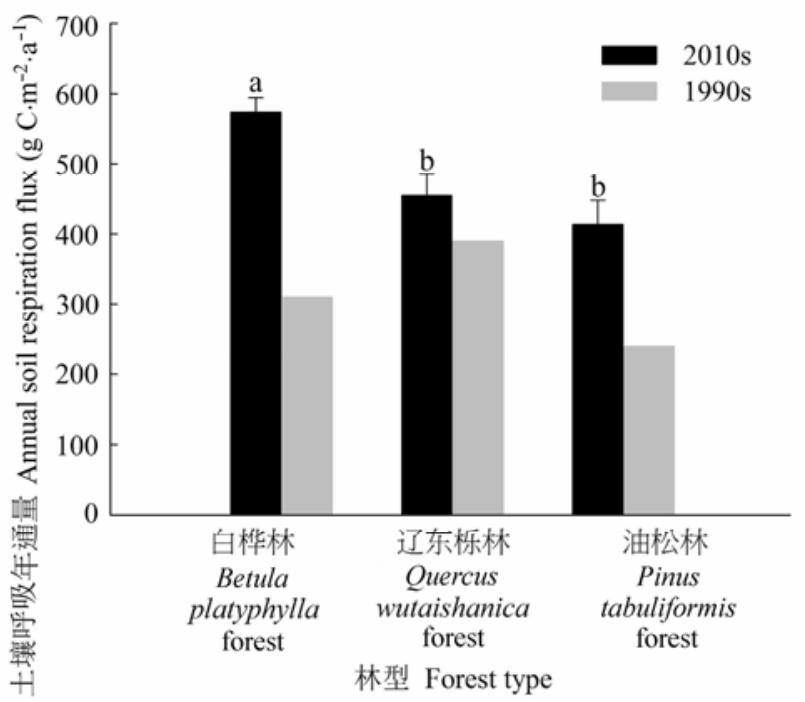

图3 北京东灵山3 种典型森林的土壤呼吸年通量。a和b表 示林分间的差异显著性 $(\alpha=0.05)$ 。

Fig. 3 Averaged annual soil respiration flux in three temperate forests on Mt. Dongling, Beijing. a and b denote significant difference among forest types at $\alpha=0.05$.

\section{2 土壤呼吸年通量 20 年的变化}

如图3所示, 过去 20 年, 3 个样地的土壤呼吸年 通量均有不同程度的增加, 增加幅度在 $17 \%-85 \%$ 之 间。以下 3 种因素可能导致这种增加。首先, 由于气 候变化导致的温度升高促进了土壤呼吸(BondLamberty \& Thomson, 2010; 苏宏新和李广起, 2012)。其次, 3 个样地的生物量在20年间持续积累: 白桦林、辽东柇林、油松林的植被生物量分别从1990 年的95.0、75.2、94.5 Mg.hm ${ }^{-2}$ 增加到2010年的198.6、 139.2、199.9 $\mathrm{Mg} \cdot \mathrm{hm}^{-2}$, 其中根系生物量从19.0、 18.8、17.0 Mg.hm ${ }^{-2}$ 增加到39.7、33.4、40.0 Mg.hm ${ }^{-2}$, 增幅达78\%-135\% (胡雪洋, 2015)。根系呼吸是土壤 呼吸的重要组成部分(Hanson et al., 2000; Shibistova et al., 2002), 根系生物量的增加显然会导致土壤呼 吸的增加。再次, 土壤呼吸的底物浓度变化也可能 导致土壤呼吸年通量的变化。土壤微生物的活动依 赖于植物地上部分和根系向土壤输入的有机物, 所 以根系分泌物的变化会导致土壤呼吸底物供应的变 化。此外, 地上和地下生物量的增加还可以导致土 壤 $\mathrm{pH}$ 值、土壤温度和水分等直接影响因子的变化, 从而引起土壤呼吸的变化 (Raich \& Schlesinger, 1992; Sitaula et al., 1995; Boone et al., 1998)。土壤呼 吸年通量的增加表明, 这3种森林在近 20 年来的碳 周转速率在显著加快。

\section{3 不确定性}

本文对东灵山3种温带森林固定样地的土壤呼 吸进行了连续3年的观测, 并与 20 世纪 90 年代的土壤 呼吸值进行了对比。在分析过程中, 以下3个因素可 能给结果带来不确定性。

\subsection{1 土壤性状的空间异质性}

森林中根系的分布、地表调落物的格局和土壤 有机质的深度等都存在很大的空间异质性，这些因 素对土壤呼吸有重要影响(Scott-Denton et al., 2006; Tang et al., 2005), 从而可能导致土壤呼吸存在较大 的空间变异。我们虽然在同一样方中对土壤呼吸进 行了复查, 但无法定位到20年前观测的具体位置, 这可能对观测结果产生影响。

\subsection{2 土壤水分}

土壤水分是影响土壤呼吸的重要因素(Bowden et al., 1993), 而我们的土壤呼吸模型仅考虑了 $5 \mathrm{~cm}$ 土壤温度这个因素。如果加入土壤水分因子, 可能会 提高我们对土壤呼吸预测的准确性。

\subsection{3 植被物候的影响}

非生长季的土壤呼吸与温度的关系不同于生长 季(Monson et al., 2006)。因此, 使用生长季土壤呼吸 速率与温度的 $Q_{10}$ 模型外推全年土壤呼吸速率可能会 给结果带来不确定性。

基金项目 国家自然科学基金(31321061和31330012)、全球变化研究国家重大科学研究计划(2014CB954001) 和中国科学院先导项目 (XDA05050000)。

致谢 中国科学院植物研究所沈海花、陈雅涵、张 建华、罗永开, 北京大学郑天立等在实验中给予支 持和帮助, 北京大学唐志尧、胡小康、井新、李鹏、 王永慧和蔡琼在论文修改校对中给予宝贵意见, 中 国科学院北京森林生态系统定位研究站桑卫国、苏 宏新、白帆等工作人员在野外实验中提供帮助, 一 并致谢!

\section{参考文献}

Anderson JM (1973). Carbon dioxide evolution from two temperate, deciduous woodland soils. Journal of Applied Ecology, 10, 361-378.

Atkin OK, Edwards EJ, Loveys BR (2000). Response of root respiration to changes in temperature and its relevance to global warming. New Phytologist, 147, 141-154.

Bond-Lamberty B, Thomson A (2010). Temperature-associated increases in the global soil respiration record. Nature, 464, 579-582. 
Boone RD, Nadelhoffer KJ, Canary JD, JP Kaye JP (1998). Roots exert a strong influence on the temperature sensitivity of soil respiration. Nature, 396, 570-572.

Bowden RD, Nadelhoffer KJ, Boone RD, Melillo JM, Garrison JB (1993). Contributions of aboveground litter, belowground litter, and root respiration to total soil respiration in a temperate mixed hardwood forest. Canadian Journal of Forest Research, 23, 1402-1407.

Bronson DR, Gower ST, Tanner M, Linder S, van Herk I (2008). Response of soil surface $\mathrm{CO}_{2}$ flux in a boreal forest to ecosystem warming. Global Change Biology, 14, 856-867.

Burton AJ, Pregitzer KS, Zogg GP, Zak DR (1998). Drought reduces root respiration in sugar maple forests. Ecological Applications, 8, 771-778.

Chang JG, Liu SR, Shi ZM, Chen BY, Zhu XL (2007). Soil respiration and its components partitioning in the typical forest ecosystems at the transitional area from the northern subtropics to warm temperate, China. Acta Ecologica Sinica, 27, 1791-1802. (in Chinese with English abstract) [常建国, 刘世荣, 史作民, 陈宝玉, 朱学凌 (2007). 北 亚热带一南暖温带过渡区典型森林生态系统土壤呼吸 及其组分分离. 生态学报, 27, 1791-1802.]

Chang ZQ, Feng Q, Si JH, Li JL, Su YH (2008). Soil carbon storage and $\mathrm{CO}_{2}$ flux under different vegetation types in Qilian Mountains. Chinese Journal of Ecology, 27, 681-688. (in Chinese with English abstract) [常宗强, 冯 起, 司建华, 李建林, 苏永红 (2008). 祁连山不同植被 类型土壤碳贮量和碳通量. 生态学杂志, 27, 681-688.]

Davidson EA, Janssens IA (2006). Temperature sensitivity of soil carbon decomposition and feedbacks to climate change. Nature, 440, 165-173.

Dörr H, Münnich KO (1987). Annual variation in soil respiration in selected areas of the temperate zone. Tellus B, 39B, 114-121.

Edwards NT, Harris WF (1977). Carbon cycling in a mixed deciduous forest floor. Ecology, 58, 431-437.

Edwards NT, Ross-Todd BM (1979). The effects of stem girdling on biogeochemical cycles within a mixed deciduous forest in eastern Tennessee. Oecologia, 40, 247-257.

Edwards NT, Ross-Todd BM (1983). Soil carbon dynamics in a mixed deciduous forest following clear-cutting with and without residue removal. Soil Science Society of America Journal, 47, 1014-1021.

Ewel KC, Cropper JWP Jr, Gholz HL (1987a). Soil CO $\mathrm{CO}_{2}$ evolution in Florida slash pine plantations. I. Changes through time. Canadian Journal of Forest Research, 17, 325-329.

Ewel KC, Cropper JWP Jr, Gholz HL (1987b). Soil CO $\mathrm{CO}_{2}$ evolution in Florida slash pine plantations. II. Importance of root respiration. Canadian Journal of Forest Research, 17, 330-333.

Fang JY, Liu GH, Zhu B, Wang XK, Liu SH (2007). Carbon budgets of three temperate forest ecosystems in Dongling Mt., Beijing, China. Science in China Series D: Earth Sciences, 50, 92-101.

Fang JY, Wang W (2007). Soil respiration as a key belowground process: Issues and perspectives. Journal of Plant Ecology (Chinese Version), 31, 345-347. (in Chinese with English abstract) [方精云, 王娓 (2007). 作为地下过程 的土壤呼吸: 我们理解了多少? 植物生态学报, 31, 345-347.]

Fang JY, Wang XK, Liu GH, Kang DM (1995). Measurement of respiration amount of trees in Quercus liaotungensis community. Acta Ecologica Sinica, 15, 235-244. (in Chinese with English abstract) [方精云, 王效科, 刘国华, 康 德梦 (1995). 北京地区辽东栋呼吸量的测定. 生态学 报, 15, 235-244.]

Fisk MC, Fahey TJ (2001). Microbial biomass and nitrogen cycling responses to fertilization and litter removal in young northern hardwood forests. Biogeochemistry, 53, 201-223.

Garrett HE, Cox GS (1973). Carbon dioxide evolution from the floor of an oak-hickory forest. Soil Science Society of America Journal, 37, 641-644.

Grogan P, Chapin III FS (1999). Arctic soil respiration: Effects of climate and vegetation depend on season. Ecosystems, 2, 451-459.

Hanson PJ, Edwards NT, Garten CT, Andrews JA (2000). Separating root and soil microbial contributions to soil respiration: A review of methods and observations. Biogeochemistry, 48, 115-146.

Högberg P, Bhupinderpal-Singh, Löfvenius MO, Nordgren A (2009). Partitioning of soil respiration into its autotrophic and heterotrophic components by means of tree-girdling in old boreal spruce forest. Forest Ecology and Management, 257, 1764-1767.

Högberg P, Nordgren A, Buchmann N, Taylor AFS, Ekblad A, Högberg MN, Nyberg G, Ottosson-Löfvenius MO, Read DJ (2001). Large-scale forest girdling shows that current photosynthesis drives soil respiration. Nature, 411, 789792.

Hu XY (2015). Carbon Cycling of Temperate Forest Ecosystems and Its Response to Nitrogen Fertilization in Mt. Dongling, Beijing. PhD dissertation, Peking University, Beijing. 25-28. (in Chinese with English abstract) [胡雪洋 (2015). 北京东灵山温带森林生态系统碳循环及其对施 氮的响应. 博士学位论文, 北京大学, 北京. 25-28.]

Johnson DW, van Hook RI (1989). Analysis of Biogeochemical Cycling Processes in Walker Branch Watershed. Springer, New York. 197-232.

Linn DM, Doran JW (1984). Effect of water-filled pore space on carbon dioxide and nitrous oxide production in tilled and nontilled soils. Soil Science Society of America Journal, 48, 1267-1272. 
Liu JJ, Wang DX, Lei RD, Wu QX (2003). Soil respiration and release of carbon dioxide from natural forest of Pinus tabulaeformis and Quercus aliena var. acuteserrata in Qinling Mountains. Scientia Silvae Sinicae, 39(2), 87-13. (in Chinese with English abstract) [刘建军, 王得祥, 雷瑞 德, 吴钦孝 (2003). 秦岭天然油松、锐齿栋林地土壤呼 吸与 $\mathrm{CO}_{2}$ 释放. 林业科学, 39(2), 78-13.]

Liu SH, Fang JY, Makoto K (1998). Soil respiration of mountainous temperate forests in Beijing, China. Acta Phytoecologica Sinica, 22, 119-126. (in Chinese with English abstract) [刘绍辉, 方精云, 清田信 (1998). 北京山地温 带森林的土壤呼吸. 植物生态学报, 22, 119-126.]

Monson RK, Lipson DL, Burns SP, Turnipseed AA, Delany AC, Williams MW, Schmidt SK (2006). Winter forest soil respiration controlled by climate and microbial community composition. Nature, 439, 711-714.

Raich JW, Potter CS (1995). Global patterns of carbon dioxide emissions from soils. Global Biogeochemical Cycles, 9, 23-36.

Raich JW, Schlesinger WH (1992). The global carbon dioxide flux in soil respiration and its relationship to vegetation and climate. Tellus B, 44, 81-99.

Rayment MB, Jarvis PG (2000). Temporal and spatial variation of soil $\mathrm{CO}_{2}$ efflux in a Canadian boreal forest. Soil Biology \& Biochemistry, 32, 35-45.

Reiners WA (1968). Carbon dioxide evolution from the floor of three Minnesota forests. Ecology, 49, 471-483.

Reinke JJ, Adriano DC, McLeod KW (1981). Effects of litter alteration on carbon dioxide evolution from a South Carolina pine forest floor. Soil Science Society of America Journal, 45, 620-623.

Savage KE, Davidson EA (2001). Interannual variation of soil respiration in two New England forests. Global Biogeochemical Cycles, 15, 337-350.

Schlesinger WH, Andrews JA (2000). Soil respiration and the global carbon cycle. Biogeochemistry, 48, 7-20.
Scott-Denton LE, Rosenstiel TN, Monson RK (2006). Differential controls by climate and substrate over the heterotrophic and rhizospheric components of soil respiration. Global Change Biology, 12, 205-216.

Shibistova O, Lloyd JON, Evgrafova S, Savushkina N, Zrazhevskaya G, Arneth A, Knohl A, Kolle O, Schulze ED (2002). Seasonal and spatial variability in soil $\mathrm{CO}_{2}$ efflux rates for a central Siberian Pinus sylvestris forest. Tellus B, 54, 552-567.

Sitaula BK, Bakken LR, Abrahamsen G (1995). N-fertilization and soil acidification effects on $\mathrm{N}_{2} \mathrm{O}$ and $\mathrm{CO}_{2}$ emission from temperate pine forest soil. Soil Biology \& Biochemistry, 27, 1401-1408.

Su HX, Li GQ (2012). Simulating the response of the Quercus mongolica forest ecosystem carbon budget to asymmetric warming. Chinese Science Bulletin, 57, 1544-1552. (in Chinese with English abstract) [苏宏新, 李广起 (2012). 模拟蒙古栋林生态系统碳收支对非对称性升温的响应. 科学通报, 57, 1544-1552.]

Tang JW, Baldocchi DD, Xu LK (2005). Tree photosynthesis modulates soil respiration on a diurnal time scale. Global Change Biology, 11, 1298-1304.

Vogt KA, Edmonds RL, Antos GC, Vogt DJ (1980). Relationships between $\mathrm{CO}_{2}$ evolution, ATP concentrations and decomposition in four forest ecosystems in western Washington. Oikos, 35, 72-79.

Wang CK, Yang JY, Zhang QZ (2006). Soil respiration in six temperate forests in China. Global Change Biology, 12, 2103-2114.

Wang W, Peng SS, Wang T, Fang JY (2010). Winter soil $\mathrm{CO}_{2}$ efflux and its contribution to annual soil respiration in different ecosystems of a forest-steppe ecotone, North China. Soil Biology \& Biochemistry, 42, 451-458.

责任编委：杨元合 责任编辑: 王 葳 